\title{
COMPARISON OF NOVEL CLINICALLY APPLICABLE METHODOLOGY FOR SENSITIVE DIAGNOSTICS OF CARTILAGE DEGENERATION
}

\author{
P Kiviranta $^{1 *}$, J Töyräs ${ }^{2}$, MT Nieminen ${ }^{3}$, MS Laasanen ${ }^{4}$, S Saarakkala ${ }^{5}$, HJ Nieminen ${ }^{1}$, MJ Nissi ${ }^{1}$, JS Jurvelin ${ }^{1,6}$ \\ ${ }^{1}$ Department of Physics, University of Kuopio, Kuopio, Finland \\ ${ }^{2}$ Department of Clinical Neurophysiology, Kuopio University Hospital and University of Kuopio, Kuopio \\ ${ }^{3}$ Department of Diagnostic Radiology, Oulu University Hospital, Oulu, Finland \\ ${ }^{4}$ Savonia Polytechnic, Engineering Kuopio, Information Technology R\&D Unit, Kuopio \\ ${ }^{5}$ Department of Nuclear Medicine, Mikkeli Central Hospital, Mikkeli, Finland \\ ${ }^{6}$ Department of Clinical Physiology and Nuclear Medicine, Kuopio University Hospital, Kuopio
}

\begin{abstract}
In order efficiently to target therapies intending to stop or reverse degenerative processes of articular cartilage, it would be crucial to diagnose osteoarthritis (OA) earlier and more sensitively than is possible with the existing clinical methods. Unfortunately, current clinical methods for OA diagnostics are insensitive for detecting the early degenerative changes, e.g., arising from collagen network damage or proteoglycan depletion. We have recently investigated several novel quantitative biophysical methods, including ultrasound indentation, quantitative ultrasound techniques and magnetic resonance imaging, for diagnosing the degenerative changes of articular cartilage, typical for OA. In this study, the combined results of these novel diagnostic methods were compared with histological (Mankin score, MS), compositional (proteoglycan, collagen and water content) and mechanical (dynamic and equilibrium moduli) reference measurements of the same bovine cartilage samples. Receiver operating characteristics (ROC) analysis was conducted to judge the diagnostic performance of each technique. Indentation and ultrasound techniques provided the most sensitive measures to differentiate samples of intact appearance $(\mathrm{MS}=0)$ from early $(1 \leq \mathrm{MS} \leq 3)$ or more advanced (MS $>3)$ degeneration. Furthermore, these techniques were good predictors of tissue composition and mechanical properties. The specificity and sensitivity analyses revealed that the mechano-acoustic methods, when further developed for in vivo use, may provide more sensitive probes for $\mathrm{OA}$ diagnostics than the prevailing qualitative X-ray and arthroscopic techniques. Noninvasive quantitative MRI measurements showed slightly lower diagnostic performance than mechano-acoustic techniques. The compared methods could possibly also be used for the quantitative monitoring of success of cartilage repair.
\end{abstract}

Key Words: Articular cartilage, osteoarthritis, patella, bovine, arthroscopy, magnetic resonance imaging, dGEMRIC, indentation, ultrasound.

*Address for correspondence:

Panu Kiviranta

Department of Physics, University of Kuopio

P.O.B 1627, 70211 Kuopio, Finland

Telephone Number ++358-17-162530

FAX Number: ++358-17-16 2585

E-mail: panu.kiviranta@helsinki.fi
Introduction

Osteoarthritis (OA) is a severe disease of the joints, in which articular cartilage is degenerated and, eventually, worn away. The early changes in cartilage tissue, associated with OA, include loss of proteoglycans (PGs) and degradation of the collagen fibril network (Buckwalter and Mankin, 1997). This leads to softening of the tissue (Armstrong and Mow, 1982). Softened articular cartilage fails to resist impact forces during normal loading and this endangers the tissue to fissures and fibrillation (Palmoski and Brandt, 1981). Tissue degeneration leads to inflow of water and, thereby, to an increase in water content of the tissue. At this stage, cartilage is even more prone to wearing. Besides degenerative changes in cartilage, the underlying bone undergoes a remodeling process that leads to a sclerosis of the subchondral bone (Radin, 1976). The developing OA increases pain, restricts exercise and limits physical capability. The earliest degenerative changes may be reversible, i.e. changing loading conditions, surgical operation or potentially pharmacological intervention may slow down or even stop the progression of OA (Buckwalter and Mankin, 1997; Freeman, 1999). When OA progresses to its terminal point, cartilage tissue is almost completely worn away exposing the subchondral bone. Currently, there is no efficient way to re-establish eroded cartilage and, therefore, only palliative treatment or arthroplasty can be used to relieve patients. Therefore, it would be crucial to recognize the very early changes of OA to target the treatments efficiently.

Traditionally, the diagnosis of osteoarthritis (OA) is based on patients' symptoms and X-ray imaging. The measurement of joint gap narrowing in X-ray images is an indirect way to assess the thickness of articular cartilage between two articulating bones. Unfortunately, the changes visible with this method represent the final OA stages. Magnetic resonance imaging (MRI) or arthroscopy are also used to evaluate the integrity of articular cartilage. However, these methods are qualitative and still lack the ability to detect the earliest degenerative changes. Further, the emergence of novel surgical methods for repairing damaged cartilage has increased the demand for sensitive assessment of the quality of repaired cartilage.

During the last few years, several clinically potential quantitative MRI, mechanical and ultrasound methods have been developed to analyze sensitively the structure, composition and functional properties of articular cartilage. The delayed gadolinium-enhanced MRI of 
cartilage (dGEMRIC) has been shown to predict the PG content of cartilage (Bashir et al., 1999, Nieminen et al., 2002), whereas the $\mathrm{T} 2$ relaxation time reflects the integrity and arrangement of the collagen fibril network (Nieminen et al., 2000; Nieminen et al., 2001; Xia et al., 2001) as well as collagen and water content (Fragonas et al., 1998; Shapiro et al., 2001). Both techniques have been applied for in vivo analysis of human cartilage using clinical MR scanners (Gold et al., 2006), and e.g. cartilage maturation after repair operation has been successfully monitored (Gillis et al., 2001).

Acoustic properties of cartilage, i.e. attenuation, speed of sound, ultrasound (US) reflection coefficient for the cartilage surface and ultrasonically determined surface roughness, have been demonstrated to change during cartilage degeneration (Cherin et al., 1998; Nieminen et al., 2004a; Saarakkala et al., 2004; Töyräs et al., 2003). US indentation, a technique that combines the determination of dynamic cartilage stiffness and measurement of US reflection coefficient for the cartilage surface, efficiently diagnosed degenerated and intact tissue, and even differentiated proteoglycan depletion and collagen disruption (Laasanen et al., 2002; Saarakkala et al., 2003). In principle, these mechano-acoustic measurements can be conducted with a single arthroscopic measurement device. Furthermore, an arthroscopic ultrasound imaging device could be developed and used to diagnose degenerative processes of cartilage tissue. The benefit of ultrasound imaging, as compared to more localized measurements, is the possibility to obtain rapidly extensive information from larger areas of articular surfaces. In addition, the estimation of cartilage surface roughness is difficult by using the localized techniques.

So far, there has been no objective comparison of the diagnostic performance of quantitative MRI (Bashir et al., 1999; Nieminen et al., 2001; Nieminen et al., 2002; Xia et al., 2002), US indentation (Laasanen et al., 2002, Suh et al., 2001) and acoustic measurements (Cherin et al., 1998; Nieminen et al., 2004a; Saarakkala et al., 2004; Töyräs et al., 2003). In this study, we combined the data from our earlier experiments with bovine cartilage samples (Nieminen et al., 2004a; Nissi et al., 2004; Saarakkala et al., 2003; Saarakkala et al., 2006; Töyräs et al., 2003) and compared the capability of these quantitative techniques to detect early degenerative changes. The results from these novel techniques were compared with histological (Mankin score), compositional (proteoglycan, collagen and water content) and mechanical (dynamic and equilibrium moduli) reference measurements. Receiver operating characteristics (ROC) analysis was conducted to judge the diagnostic performance of each technique.

\section{Methods}

Detailed description of the materials and methods for ultrasound, mechanical and MRI measurements and analyses are presented in our previous papers (Laasanen et al., 2003b; Nieminen et al., 2004a; Nissi et al., 2004; Saarakkala et al., 2003; Saarakkala et al., 2006; Töyräs et

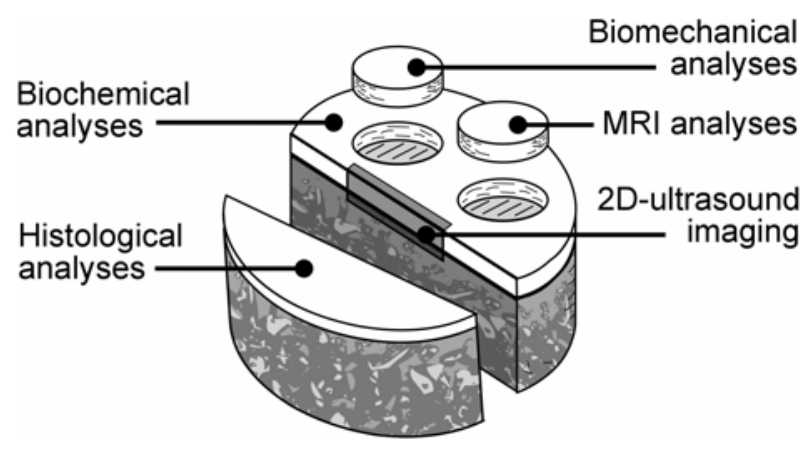

Figure 1. Schematic presentation of sample preparation. Osteochondral plugs $(\varnothing=19 \mathrm{~mm})$ from bovine patella were cut into pieces that were analysed using novel diagnostic and reference techniques.

al., 2003). Therefore, only a brief summary of methods is presented.

\section{Sample preparation}

The outline of sample processing is presented in Fig. 1. Osteochondral plugs (diameter $19 \mathrm{~mm}$ ) with healthy and spontaneously degenerated cartilage were prepared from fresh bovine patellae ( $n=32)$ (Saarakkala et al., 2003). The samples were classified visually into four categories: cartilage with intact surface (group $1, n=11$ ), cartilage with smooth surface but slight colouration (group 2, $n=5$ ), cartilage with superficial damage (group $3, n=6$ ) and cartilage with deep defects (group $4, n=8$ ). The samples were frozen in phosphate buffered saline (PBS) for later measurements.

\section{Ultrasound measurements}

A commercial ultrasound 2D imaging instrument (Dermascan-C, Cortex Ltd., Hadsund, Denmark) was used to image the osteochondral samples in-situ and, subsequently, Ultrasound Roughness Index $(U R I, \mu \mathrm{m})$ and Integrated Reflection Coefficient (IRC, dB) were determined for the cartilage surface, as described earlier (Saarakkala et al., 2004). Further, in situ local measurements were performed with US indentation instrument (Saarakkala et al., 2003; Saarakkala et al., 2006) to determine cartilage dynamic modulus $\left(E_{\text {dyn }}, \mathrm{MPa}\right)$ and ultrasound reflection coefficient for the cartilage surface $(R, \%)$ (Fig. 2a). Subsequently, cartilage layer was isolated using a dermal biopsy punch $(\varnothing=4 \mathrm{~mm})$ and a razor blade. Using a mechano-acoustic tester (Töyräs et al., 2003) integrated US attenuation ( $\mathrm{dB} / \mathrm{mm}, 5-9 \mathrm{MHz})$, speed of sound $(\mathrm{m} / \mathrm{s})$, reference equilibrium $\left(E_{\text {ref }}, \mathrm{MPa}\right)$ and dynamic (instantaneous) moduli $\left(E_{\text {dyn,ref }}, \mathrm{MPa}\right)$ were determined in unconfined compression geometry (Nieminen et al., 2004a) (Fig. 2b).

\section{MRI measurements}

MRI parameters were determined with a $9.4 \mathrm{~T}$ Oxford 400 NMR vertical magnet at $25^{\circ} \mathrm{C}$. The $\mathrm{T} 2$ relaxation times were determined while the sample was immersed in PBS. For dGEMRIC measurements the samples were balanced in $1 \mathrm{mM} \mathrm{Gd-DTPA}{ }^{2-}$ (Magnevist, Schering, Berlin, 
Germany) solution for a minimum of 2.5 hours and T1 relaxation times in presence of Gd-DTPA ${ }^{2-}(T 1 G d)$ were determined. From MRI analyses, the superficial value of T2 (T2) and bulk dGEMRIC values (T1Gd) were selected for this study (Nissi et al., 2004).

\section{Histological analyses}

Mankin score (Mankin et al., 1971) of the samples was evaluated by three investigators from safranin-O stained blind coded sections and averaged value was used for analysis. Samples were divided into three categories depending on the total score. Mankin score 0 indicated intact cartilage $(n=11)$, scores from 1-3 represented early degeneration $(n=11)$ and score over 3 indicated advanced degeneration $(n=10)$ (Nieminen et al., 2004a).

\section{Biochemical analyses}

Water content of the samples was measured by weighing the samples before and after freeze-drying (Töyräs et al., 2003). To determine PG concentration of the samples, uronic acid content was quantified from GAGs extracted from the samples and normalized to the wet weight of the tissue. Hydroxyproline content was determined to quantify the collagen content of the tissue with a spectrophotometric assay after the hydrolysis of freeze-dried tissue.

\section{Statistical analyses}

The Kruskal-Wallis $\mathrm{H}$ test was utilized to determine the significance of differences between groups with variable degree of degeneration. The Mann-Whitney U test was conducted to determine the significance of differences between the healthy group and the group pooled from degenerated samples. For correlations with Mankin score, Spearman's rho was calculated, whereas for other correlations, Pearson's correlation coefficient was used. ROC curve analysis was conducted to find a cut off value for each parameter where the sum of specificity and sensitivity values was maximal. All statistical analyses were conducted using SPSS statistical software (version 11.0.2, SPSS inc., Chicago, IL, USA)

\section{Results}

Typical histological, 2D ultrasound and MRI images from different groups of degeneration are presented in Fig. 3. It was seen that the histologically determined cartilage degeneration level (Mankin score) was only weakly predictable by visual evaluation of cartilage surface (Fig. 4). There was no statistically significant variation in Mankin scores between the visually evaluated groups 1 and 2 (Mann-Whitney U-test, $p=0.39$ ). All acoustic, mechanical and MRI parameters showed significant variation $(p<0.01$, Kruskal-Wallis $H$ test) between the samples with different stages of degeneration (Table 1). When the samples were divided into healthy (Mankin score $=0$ ) and degenerated (Mankin score $>0$ ) ones, measured parameters revealed significant differences between groups $(p<0.01$, Mann Whitney U-test).

The diagnostic acoustic and mechanical parameters revealed high specificity and sensitivity values (Table 1).

\section{a) Ultrasound indentation}

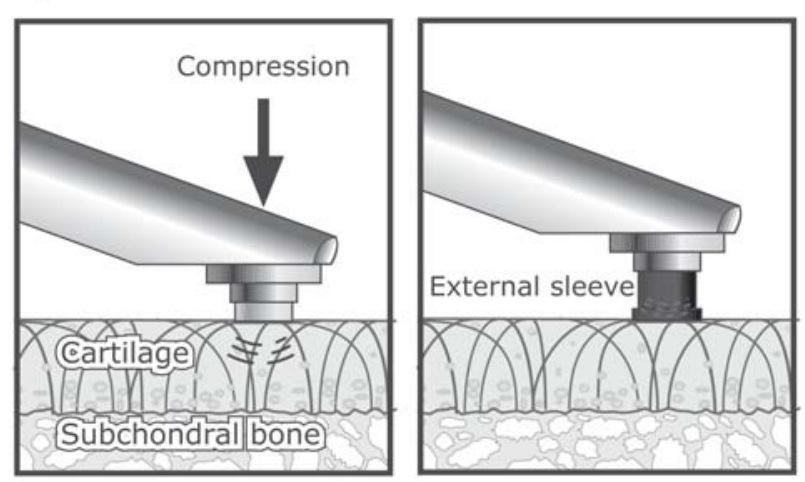

b)

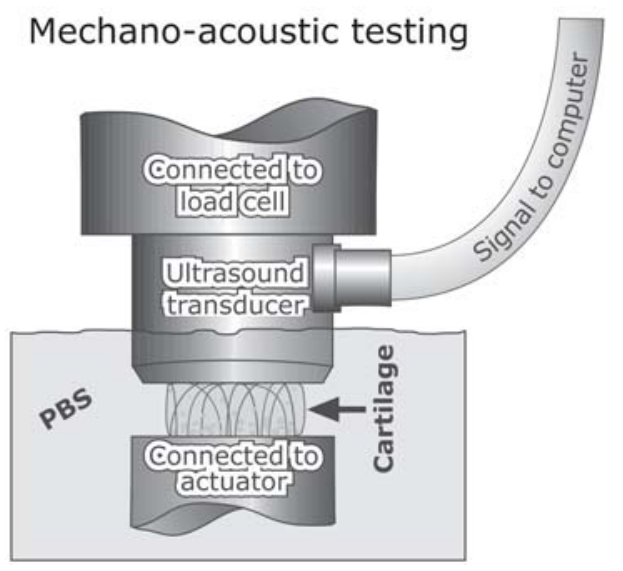

Figure 2. a) Ultrasound indentation technique. Cartilage is compressed with an ultrasound transducer mounted on an arthroscopic indentation tool to determine dynamic modulus of cartilage. When an external sleeve is applied on the transducer to keep the distance between the transducer and cartilage surface constant, coefficient of ultrasound backscattering from the superficial cartilage gives information about the smoothness of cartilage surface and collagen fibril network. b) Mechano-acoustic testing device with the ultrasound transducer was used to determine mechanical $\left(E_{r e f}, E_{d y n, r e f}\right)$ and acoustic properties (attenuation, speed of sound) of cartilage samples.

The greatest sum of sensitivity and specificity was found for the cartilage surface roughness parameter (URI, sensitivity 0.91 and specificity 1.00). The speed of sound parameter was able to recognize damaged cartilage with the sensitivity of 0.91 and specificity of 0.82 . For MRI parameters $T 1 G d$ and $T 2$ specificity and sensitivity values were slightly lower, being 0.81 for sensitivity and 0.73 for specificity.

Several novel biophysical parameters revealed strong correlations with the reference methods (Table 2). Correlation with Mankin score was highest for the US reflection parameter for cartilage surface $R(r=-0.85)$, as quantified with the ultrasound indentation instrument, and 
a) Healthy cartilage Mankin score 0
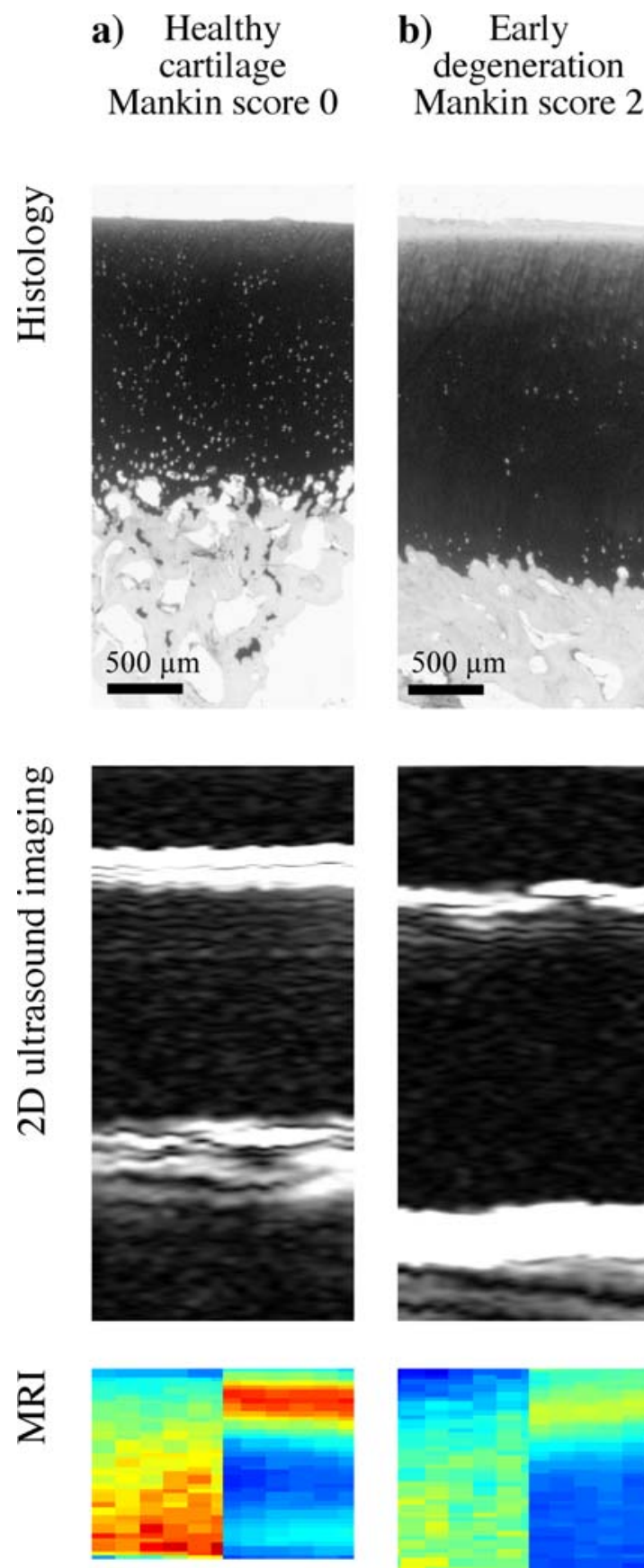
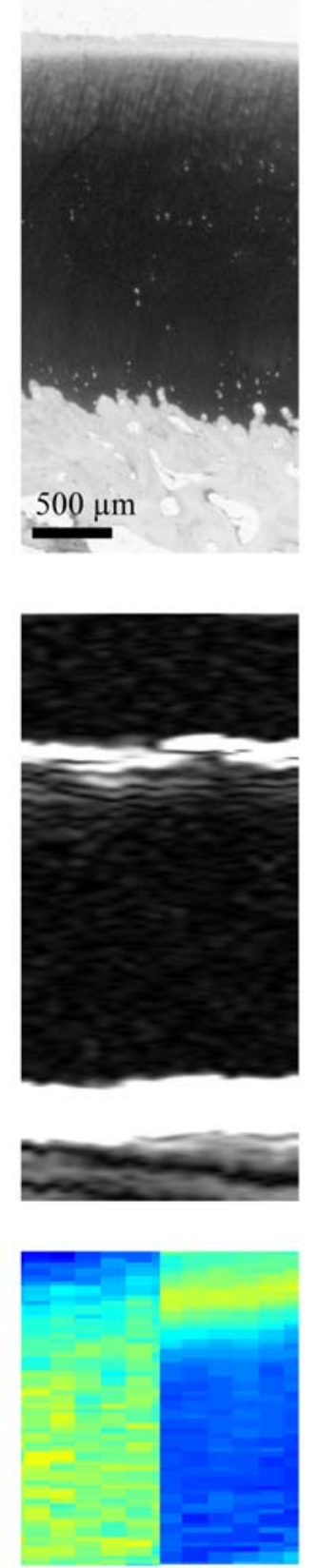

c) Advanced degeneration Mankin score 10
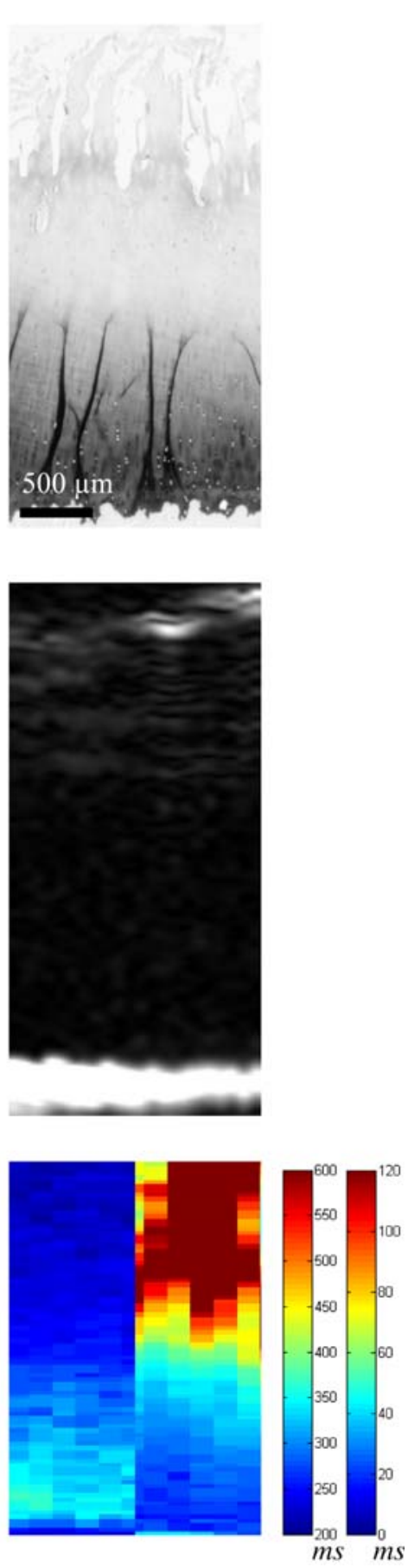

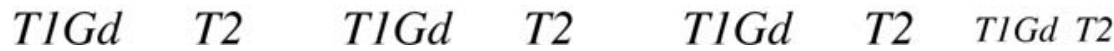

Figure 3. Microscopic digital densitometry images of Safranin-O stained cartilage sections representing different stages of cartilage degeneration and images from 2D ultrasound and quantitative MRI of the same samples. (a) Healthy cartilage. Cartilage surface is smooth and safranin-O staining is intense. The 2D ultrasound imaging shows smooth cartilage surface and a typical backscattering signal from bone. In magnetic resonance images, the sample exhibits long $T 1$ relaxation time in deep cartilage and organized structure in $T 2$ image. (b) Early degeneration. Cartilage surface is irregular and safranin-O staining slightly reduced in superficial cartilage. The surface of cartilage is irregular in the 2D ultrasound image and the cartilage bone interface shows a more intense signal. T1 relaxation is faster in deep cartilage and the superficial T2 signal signifies less oriented surface. (c) Advanced degeneration. Cartilage shows clefting to radial zone, Safranin-O staining is severely reduced. The 2D ultrasound signal from cartilage surface is low and disoriented. Cartilage surface is weak and cartilage-bone interface shows an accentuated signal. T1 relaxation is very low and superficial zone cannot be separated from PBS with gadolinium contrast agent. The fibrillated surface can be detected in the $T 2$ map. 
Table 1. Mean \pm SD values of the measured parameters from cartilage samples categorized by Mankin scoring ( 0 , $1-3,>3$ ). Cut-off, specificity and sensitivity values are calculated for diagnostic methods to discern healthy (Mankin score $=0$ ) and histologically degenerated (Mankin score $>0$ ) samples.

\begin{tabular}{|c|c|c|c|c|c|c|}
\hline \multirow[b]{2}{*}{ Reference methods } & \multicolumn{3}{|c|}{ Mankin score } & & & \\
\hline & $\begin{array}{c}\mathbf{0} \\
(\mathrm{n}=11)\end{array}$ & $\begin{array}{c}\mathbf{1 - 3} \\
(\mathrm{n}=11)\end{array}$ & $\begin{array}{c}\mathbf{3 - 1 0} \\
(\mathrm{n}=10)\end{array}$ & & & \\
\hline Thickness $(\mu \mathrm{m}) *$ & $1610 \pm 371$ & $1702 \pm 265$ & $1945 \pm 471$ & & & \\
\hline$E_{r e f}(\mathrm{MPa})^{*}$ & $0.32 \pm 0.15$ & $0.26 \pm 0.13$ & $0.08 \pm 0.07$ & & & \\
\hline$E_{\text {dyn,ref }}(\mathrm{MPa})^{*}$ & $7.06 \pm 4.83$ & $2.12 \pm 1.58$ & $0.54 \pm 0.37$ & & & \\
\hline Water $(\%)^{*}$ & $79.9 \pm 2.4$ & $81.6 \pm 1.2$ & $84.1 \pm 2.6$ & & & \\
\hline Collagen $(\mu \mathrm{g} / \mathrm{mg})^{*}$ & $10.9 \pm 3.4$ & $8.5 \pm 1.7$ & $6.7 \pm 1.0$ & & & \\
\hline PG $(\mu \mathrm{g} / \mathrm{ml})^{*}$ & $10.2 \pm 3.5$ & $6.7 \pm 1.5$ & $4.1 \pm 1.2$ & & & \\
\hline Ultrasound indentation & & & & Cut off & Specificity & Sensitivity \\
\hline$E_{d y n}(\mathrm{MPa})^{*}$ & $9.1 \pm 6.1$ & $3.7 \pm 3.6$ & $1.5 \pm 0.5$ & 2.5 & 0.89 & 0.71 \\
\hline$\underline{R(\%) *}$ & $3.7 \pm 1.2$ & $1.9 \pm 1.1$ & $0.6 \pm 0.3$ & 2.3 & 0.89 & 0.91 \\
\hline \multicolumn{7}{|l|}{ MRI } \\
\hline $\mathrm{T} 2(\mathrm{~ms})^{*}$ & $43 \pm 14$ & $69 \pm 59$ & $120 \pm 105$ & 44 & 0.73 & 0.81 \\
\hline T1GD (ms)* & $401 \pm 4$ & $371 \pm 35$ & $322 \pm 64$ & 386 & 0.73 & 0.81 \\
\hline \multicolumn{7}{|l|}{ Ultrasound } \\
\hline Attenuation $(\mathrm{dB} / \mathrm{mm})^{*}$ & $2.65 \pm 0.58$ & $2.01 \pm 0.45$ & $1.76 \pm 0.43$ & 2.46 & 0.82 & 0.95 \\
\hline Speed of sound $(\mathrm{m} / \mathrm{s})^{*}$ & $1603 \pm 27$ & $1572 \pm 15$ & $1548 \pm 14$ & 1576 & 0.82 & 0.91 \\
\hline $\operatorname{IRC}(\mathrm{dB})^{*}$ & $-26.7 \pm 1.6$ & $-30.9 \pm 4.0$ & $-37.6 \pm 4.6$ & -29.0 & 0.91 & 0.86 \\
\hline$U R I(\mu \mathrm{m}) *$ & $7.4 \pm 1.2$ & $15.1 \pm 8.5$ & $34.3 \pm 15.4$ & 10.2 & 1.00 & 0.91 \\
\hline
\end{tabular}

for surface reflection parameter IRC $(r=-0.84, p<0.01)$ and surface roughness parameter $U R I(r=0.84)$, as measured with the ultrasound imaging device. The best predictor of mechanical properties, as assessed with dynamic modulus of cartilage, was $E_{\text {dyn }}(r=0.98, p<0.01)$, as measured with the ultrasound indentation instrument. Furthermore, speed of sound was strongly associated with $E_{\text {dyn,ref }}$ and $E_{\text {ref }}$ $\left(r=0.90\right.$ and $r=0.79$, respectively, $p<0.01$ ). Overall, $E_{\mathrm{dyn}}$ and speed of sound measurements showed the highest correlation coefficients with the reference parameters.

Several diagnostic parameters were interrelated with each other (Fig. 5). $E_{\text {dyn }}$ was found to be positively interrelated with speed of sound measurements $(r=0.89$, $n=30, p<0.01)$. URI and $R$ showed negative correlation ( $r=-0.68, n=30, p<0.01)$. T1Gd and speed of sound were positively correlated $(r=0.58, n=32, p<0.01)$. Superficial $T 2$ and $U R I$ were positively interrelated $(r=0.55, n=32$, $p<0.01)$.

\section{Discussion}

More accurate and sensitive quantitative methods are needed for diagnosing the early stages of OA, as well as to evaluate the success of surgical cartilage repairs. In this study, in situ and in vitro measurements of cartilage with several novel clinically applicable methods were compared with well established reference techniques. The methods utilized in this study differ considerably from each other in their physical operation principle as well as in the potential clinical usage. Mechano-acoustic techniques necessitate arthroscopic approach while quantitative MRI imaging provides a totally non-invasive means of examination. These novel non-destructive biophysical methods showed promising potential for the diagnostics of early OA.

The high correlation coefficients with the reference parameters indicate that $E_{\text {dyn }}$ measurements provide extensive information about the mechanical properties and composition of cartilage. In particular, US indentation results were highly associated with the histological, biochemical and biomechanical integrity of the tissue, providing essential information about functional capability of cartilage. Ultrasound indentation instrument accurately determined dynamic compressive stiffness of cartilage. This method also classified the samples sensitively and specifically into normal and degenerated ones. The US reflection measurements, possible with the same instrument, were also strongly related to reference measurements. Importantly, the US indentation technique is feasible for arthroscopic measurements and could be further developed for use during routine arthroscopic procedures to screen the cartilage integrity. It could provide information about a possible decrease in cartilage stiffness, as well as about increased surface fibrillation, both of which have been shown to take place during degenerative process. This information could be used when evaluating the need for preventive treatments against OA.

In the present study, speed of sound in cartilage was found to be an effective parameter for evaluation of cartilage integrity. Speed of sound correlated highly with the structural parameters of cartilage as measured by 
Table 2. Several novel biophysical parameters revealed strong correlations with the reference methods. Correlation with Mankin score was highest for $R$ and for $I R C$ and $U R I(r=0.84)$, as measured with the $2 \mathrm{D}$ ultrasound imaging device. The best predictor of mechanical properties was $E_{\mathrm{dyn}}$, as measured with the ultrasound indentation instrument. Furthermore, speed of sound was strongly associated with $E_{\text {dyn,ref }}$ and $E_{\text {ref }}$

\section{Reference parameters}

\begin{tabular}{rccccccc}
\cline { 2 - 7 } & $\begin{array}{c}\text { Mankin } \\
\text { score }\end{array}$ & $\begin{array}{c}\text { PG } \\
\text { content }\end{array}$ & $\begin{array}{c}\text { Collagen } \\
\text { content }\end{array}$ & $\begin{array}{c}\text { Water } \\
\text { content }\end{array}$ & $\boldsymbol{E}_{\text {ref }}$ & $\boldsymbol{E}_{\text {dyn,ref }}$ & $|\boldsymbol{r}|$ \\
\hline $\boldsymbol{E}_{\text {dyn }}$ & $-0.73^{* *}$ & $0.87^{* *}$ & $0.86^{* *}$ & $-0.69^{* *}$ & $0.60^{* *}$ & $0.98^{* *}$ & 0.79 \\
$\boldsymbol{R}$ & $-0.85^{* *}$ & $0.83^{* *}$ & $0.78^{* *}$ & $-0.66^{* *}$ & $0.58^{* *}$ & $0.87^{* *}$ & 0.76 \\
US attenuation & $-0.55^{* *}$ & $0.65^{* *}$ & $0.64^{* *}$ & $-0.65^{* *}$ & $0.47^{* *}$ & $0.62^{* *}$ & 0.60 \\
Speed of sound & $-0.76^{* *}$ & $0.90^{* *}$ & $0.86^{* *}$ & $-0.80^{* *}$ & $0.79^{* *}$ & $0.90^{* *}$ & 0.83 \\
$\boldsymbol{T}_{\boldsymbol{2}}$ & $0.61^{* *}$ & $-0.36^{*}$ & -0.33 & 0.34 & -0.33 & -0.30 & 0.37 \\
$\boldsymbol{T}_{\mathbf{1 G d}}$ & $-0.54^{* *}$ & $0.62^{*}$ & 0.36 & $-0.56^{* *}$ & $0.63^{* *}$ & $0.50^{* *}$ & 0.54 \\
$\boldsymbol{I R C}$ & $-0.84^{* *}$ & $0.61^{* *}$ & $0.54^{* *}$ & $-0.48^{* *}$ & $0.58^{* *}$ & $0.58^{* *}$ & 0.61 \\
$\boldsymbol{U R I}$ & $0.84^{* *}$ & $-0.49^{* *}$ & $-0.49^{* *}$ & $0.37^{*}$ & $-0.49^{* *}$ & $-0.48^{* *}$ & 0.53 \\
\hline
\end{tabular}

$* p<0.05, * * p<0.01, n=30-32$.

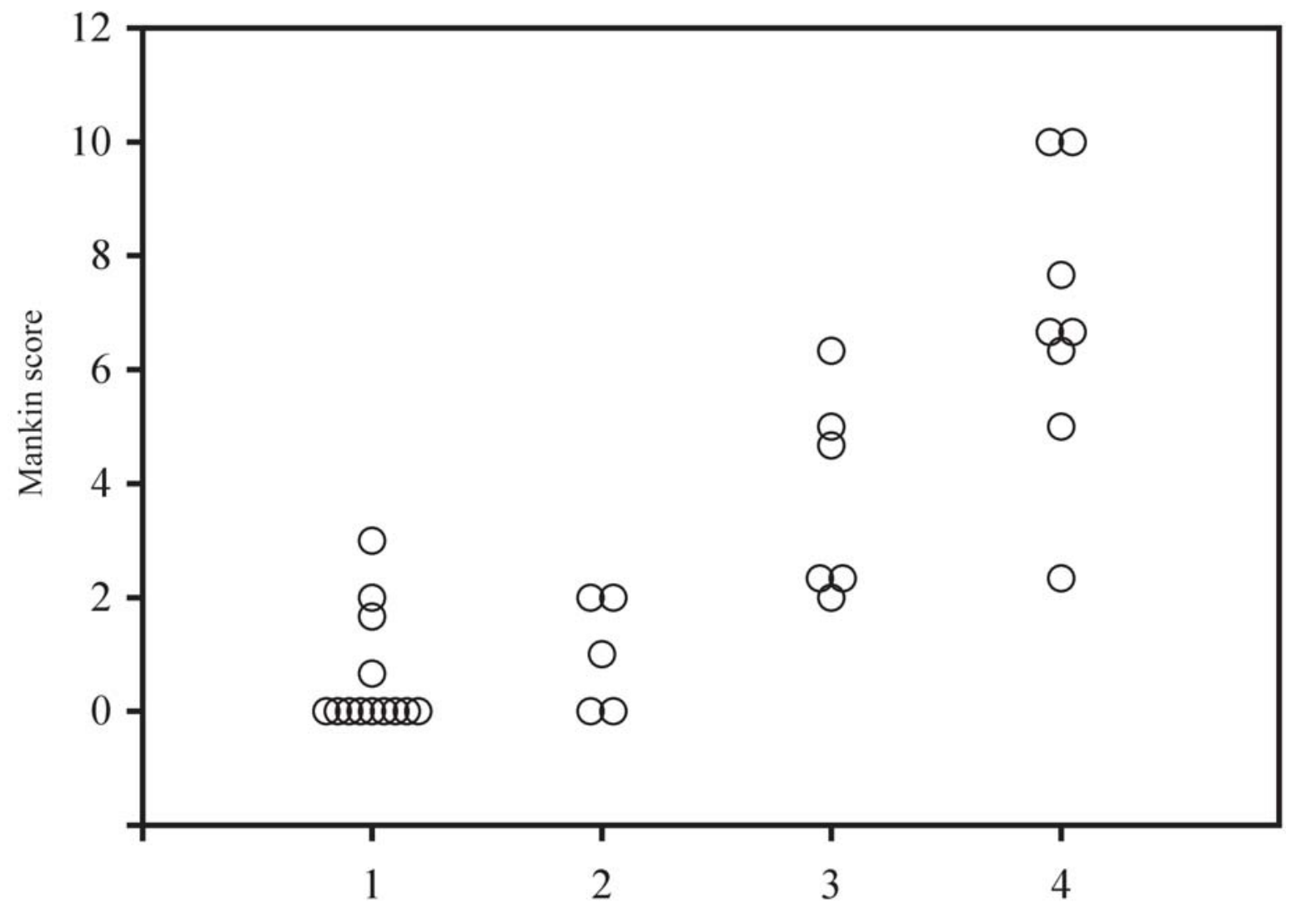

Visual grading

Figure 4. Visual grading vs. Mankin score of the cartilage samples $(n=32)$. The cartilage samples were visually graded into four categories (group 1 = intact surface; group $2=$ slight colouration but smooth surface; group $3=$ superficial damage; group 4 = deep defect, X-axis). Tissue degeneration was evaluated using the Mankin score method (Y-axis). As seen from the plot, visual evaluation of cartilage quality is not a reliable predictor of histologically assessed cartilage degeneration, especially in early degeneration. 

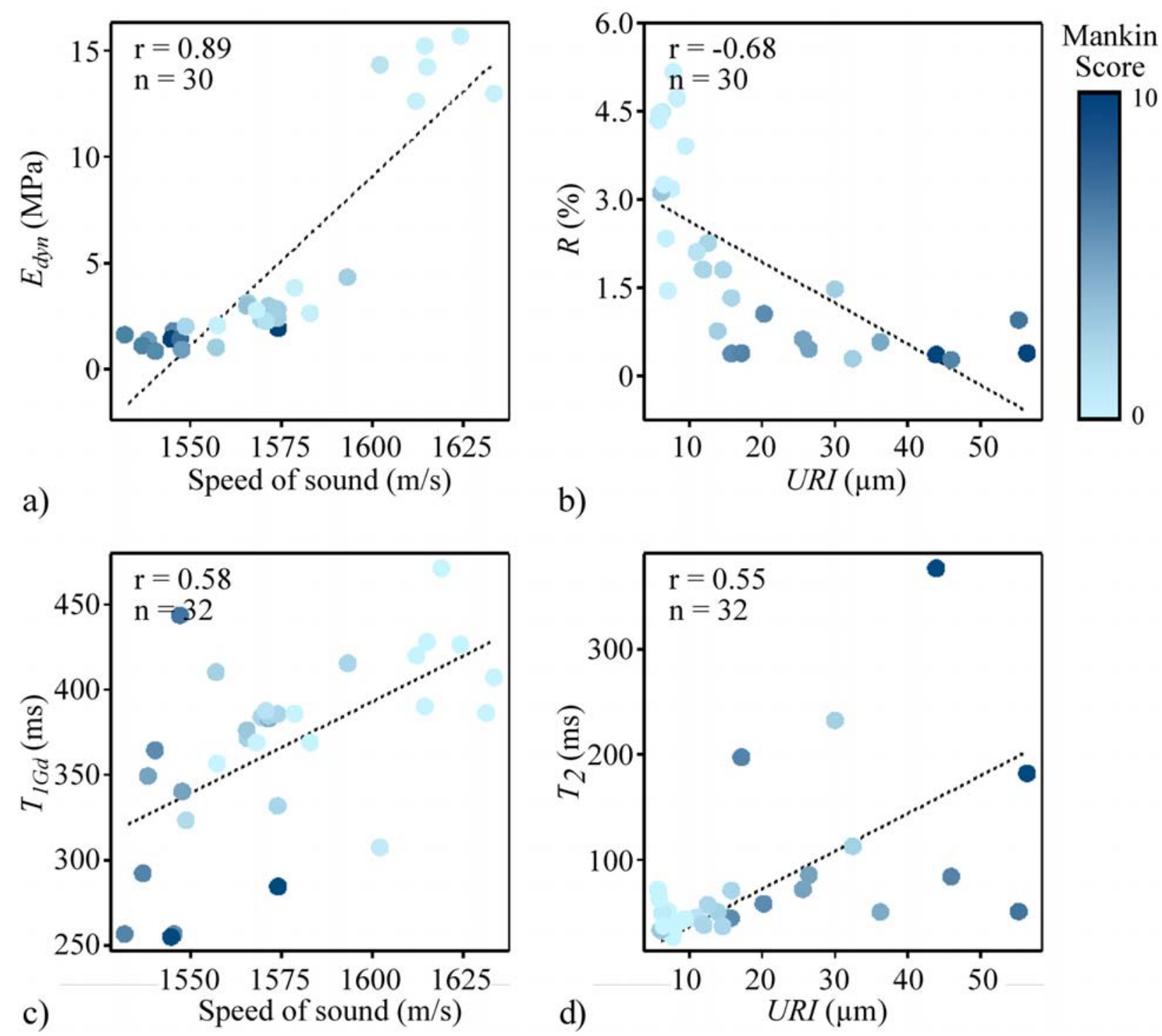

Figure 5. Scatter plots with linear fitted curves and Pearson correlation coefficients between several diagnostic parameters. The correlations were statistically significant $(p<0.01)$. Coloring indicates Mankin scores of the samples. a) Damaged samples show a systematically low dynamic modulus as measured with the US indentation. b) Ultrasound backscattering coefficient and URI give information about cartilage surface fibrillation and they are negatively interrelated. c) $T 1 G d$ and speed of sound have been shown to predict cartilage PG content. They are also positively interrelated. d) Superficial T2 values and URI are both indicators of cartilage surface integrity. The healthy samples are all situated in the lower right corner.

collagen and PG content. In addition, sensitivity and specificity of this method in distinguishing healthy and degenerated samples was good. Unfortunately, measurement of acoustic properties of articular cartilage i.e., attenuation and speed of sound, is challenging in vivo, and at the moment there are no devices for use in in vivo studies. Possibly, speed of sound measurements may be carried out during arthroscopy, as suggested earlier (Suh et al., 2001).

Parameters from quantitative 2D ultrasound imaging, i.e., IRC and URI for the cartilage surface, reflect the superficial changes that take place in cartilage during OA process. As highlighted, especially URI seems to be highly sensitive and specific for even the very earliest OA changes of tissue surface. These changes of surface roughness can not be visually detected during routine arthroscopy (Saarakkala et al., 2006). The significant associations of $U R I$ and IRC with tissue PG and collagen content as well as with the cartilage mechanical properties suggest that these properties are intertwined with each other and take place together during OA process. The methods for assessing cartilage surface integrity, i.e., URI, R and T2, were also found to be strongly interrelated, indicating that the methods are capable of detecting changes that take place simultaneously. The development of arthroscopic imaging instrument would allow real time evaluation of cartilage surface as well as cartilage-bone interface. With this method it is possible to evaluate changes below the cartilage surface not visible arthroscopically. An optimal arthroscopic tool could possibly combine indentation 
Table 3. Advantages and challenges of the novel quantitative methodology for sensitive diagnostics of osteoarthritis

\begin{tabular}{|c|c|c|c|}
\hline Method & $\begin{array}{l}\text { Sensitivity } \\
\text { Specificity }\end{array}$ & Advantages & Challenges \\
\hline $\mathrm{X}$-rays & $\begin{array}{c}- \\
+++\end{array}$ & $\begin{array}{l}\text { Non-invasive, easy to perform } \\
\text { Simultaneous bone diagnostics } \\
\text { Cheap } \\
\text { Good availability }\end{array}$ & $\begin{array}{l}\text { No direct information on cartilage } \\
\text { Poor sensitivity } \\
\text { Radiation exposure }\end{array}$ \\
\hline Quantitative MRI & $\begin{array}{l}++ \\
++\end{array}$ & $\begin{array}{l}\text { Non-invasive } \\
\text { No radiation exposure } \\
\text { Information on cartilage PGs }(T 1 G d) \\
\text { and collagen }(T 2) \\
\text { Simultaneous diagnostics of other soft } \\
\text { tissues }\end{array}$ & $\begin{array}{l}\text { Expensive } \\
\text { Poor availability }\end{array}$ \\
\hline $\begin{array}{l}\text { Ultrasound } \\
\text { indentation }\end{array}$ & $\begin{array}{l}+++ \\
+++\end{array}$ & $\begin{array}{l}\text { Can be performed during routine } \\
\text { arthroscopic procedures } \\
\text { Direct information on cartilage } \\
\text { function }\end{array}$ & $\begin{array}{l}\text { Minimally invasive } \\
\text { In vivo measurement of certain } \\
\text { locations can be challenging }\end{array}$ \\
\hline $\begin{array}{l}\text { Ultrasound } \\
\text { attenuation, speed } \\
\text { of sound }\end{array}$ & $\begin{array}{l}++ \\
++\end{array}$ & $\begin{array}{l}\text { Changes associate strongly with } \\
\text { several determinants of cartilage } \\
\text { integrity }\end{array}$ & $\begin{array}{l}\text { Lack of clinically applicable } \\
\text { measurement devices }\end{array}$ \\
\hline $\begin{array}{l}\text { 2D ultrasound } \\
\text { analysis of } \\
\text { cartilage surface }\end{array}$ & $\begin{array}{l}+++ \\
+++\end{array}$ & $\begin{array}{l}\text { Changes in cartilage surface seem to } \\
\text { reflect alterations in cartilage quality as } \\
\text { well as mechanical and compositional } \\
\text { properties of cartilage } \\
\text { Initial degeneration alters these } \\
\text { parameters }\end{array}$ & $\begin{array}{l}\text { At the moment no arthroscopic } \\
\text { instrument exists } \\
\text { Requires at least minimally invasive } \\
\text { approach }\end{array}$ \\
\hline
\end{tabular}

instrumentation with US imaging or, alternatively, clinical imaging might be carried out with a separate instrument using a high-resolution US microarray probe. However, at this point, the size and cost of such US probes set down high technical challenges before arthroscopic imaging instrument could be introduced.

MRI is a non-invasive method of imaging intraarticular changes in cartilage. The modern non-invasive MRI applications, i.e. T2 and dGEMRIC, have provided quantitative measures to evaluate the collagen fibril network organization and PG content of cartilage tissue, respectively. Although MRI showed somewhat lower correlation coefficients with the reference parameters, as compared to the mechano-acoustic techniques, specificity and sensitivity values suggested relatively good diagnostic performance for MRI. In this material, T1Gd was able to predict the total proteoglycan content of cartilage and the mechanical properties, consistently with earlier studies (Bashir et al., 1999; Nieminen et al., 2002). Especially, the concurrent measurement of both T2 and dGEMRIC in a single study is likely to improve diagnostic efficiency of MRI (Nieminen et al., 2004b). While the inferior imaging resolution of in vivo MRI may affect the sensitivity to local cartilage changes and varying field strength may affect the results, recent results with clinical MRI scanners, even with a lower resolution, are encouraging and reveal the in vivo potential of the quantitative MRI techniques (Lammentausta et al., 2006).

The good sensitivity and specificity values revealed in this study are promising. However, those numbers are preliminary and should be considered with care as the number of samples was relatively low $(n=32)$, Further, the values of intact and degenerated samples overlapped in most parameters and the normal (reference) values, i.e. Mean $\pm 2 \mathrm{SD}$, could not be applied as too many degenerated samples would have been classified into the category of intact samples. The presented cut off values suggest an optimal value which could be used to classify diagnostically bovine patellar cartilage samples. It is known that articular cartilage shows site-dependent variations in its mechanical (Laasanen et al., 2003a), acoustic (Laasanen et al., 2005) and compositional properties (Froimson et al., 1997; Kiviranta et al., 2006). Also, alteration of MRI parameter values within different joint surfaces exists (Nieminen et al., 2004b). This variation should be further investigated, and taken into account when establishing reference values for these biophysical parameters.

In this study, cartilage samples were presumed to demonstrate a uniform appearance within the $19 \mathrm{~mm}$ diameter cartilage plug (Fig. 1). Subsequently, the correlation of diagnostic method results with histological 
and biochemical properties could be established. An optimal way of assessing the degree of OA can be debated (Pritzker et al., 2006). In this study, we utilized the traditional Mankin score to judge structural integrity of cartilage. Thereby, the samples in intact cartilage group were verified histologically to be undamaged. The group of early degeneration consisted of samples with some signs of degeneration, however, this cartilage was still likely functionally nearly normal with no symptoms. The rest of the samples were classified into the group of advanced degeneration. This category demonstrated the greater variation in histological degeneration. The present study included no samples from areas of complete cartilage denudation, i.e. no samples exhibited alterations typical of end stage OA. Obviously, all methods presented in this study are capable of detecting the absence of articular cartilage. However, this stage can also be detected from $\mathrm{X}$-ray images as well as visually during artroscopic procedures, and the main benefit of these quantitative novel methods with high sensitivity and specificity is obtained when diagnosing early OA changes.

In summary, the development of novel biophysical methods provides tools for detecting early degenerative signs of cartilage, potentially also in clinical practice. The potentials and challenges of these methods are summarized in Table 3. MRI has been shown to predict structural and compositional properties of cartilage. Its non-invasiveness makes it possible to use this method for screening of this condition, when potential treatment methods have evolved. The US indentation method requires an arthroscopic procedure to be conducted. However, arthroscopy is a minimally invasive method and in routine use as millions of procedures are conducted annually anyhow. Measurement of US indentation parameters or ultrasound imaging during standard procedures could give crucial information to target possible treatments for patients. However, more research on human tissue, as well as technical development, is required to confirm these promising findings in vivo.

\section{Acknowledgements}

Financial support from Instrumentarium Science Foundation and the Academy of Finland (project 205886) is acknowledged.

\section{References}

Armstrong CG, Mow VC (1982) Variations in the intrinsic mechanical properties of human articular cartilage with age, degeneration, and water content. J Bone Joint Surg Am 64: 88-94.

Bashir A, Gray ML, Hartke J, Burstein D (1999) Nondestructive imaging of human cartilage glycosaminoglycan concentration by MRI. Magn Reson Med 41: 857-865.

Buckwalter JA, Mankin HJ (1997) Articular Cartilage, Part II: Degeneration and osteoarthritis, repair, regeneration, and transplantation. J Bone Joint Surg Am 79: 612-632.

Cherin E, Saied A, Laugier P, Netter P, Berger G (1998) Evaluation of acoustical parameter sensitivity to agerelated and osteoarthritic changes in articular cartilage using 50-MHz ultrasound. Ultrasound Med Biol 24: 341354.

Fragonas E, Mlynarik V, Jellus V, Micali F, Piras A, Toffanin R, Rizzo R, Vittur F (1998) Correlation between biochemical composition and magnetic resonance appearance of articular cartilage. Osteoarthritis Cartilage 6: $24-32$.

Freeman MA (1999) Is collagen fatigue failure a cause of osteoarthrosis and prosthetic component migration? A hypothesis. J Orthop Res 17: 3-8.

Froimson MI, Ratcliffe A, Gardner TR, Mow VC (1997) Differences in patellofemoral joint cartilage material properties and their significance to the etiology of cartilage surface fibrillation. Osteoarthritis Cartilage 5: 377-386.

Gillis A, Bashir A, McKeon B, Scheller A, Gray ML, Burstein D (2001) Magnetic resonance imaging of relative glycosaminoglycan distribution in patients with autologous chondrocyte transplants. Invest Radiol 36: 743-748.

Gold GE, Burstein D, Dardzinski B, Lang P, Boada F, Mosher T (2006) MRI of articular cartilage in OA: novel pulse sequences and compositional/functional markers. Osteoarthritis Cartilage 14: 76-86.

Kiviranta P, Rieppo J, Korhonen RK, Julkunen P, Töyräs J, Jurvelin JS (2006) Collagen network primarily controls Poisson's ratio of bovine articular cartilage in compression. J Orthop Res 24: 690-699.

Laasanen MS, Töyräs J, Hirvonen J, Saarakkala S, Korhonen RK, Nieminen MT, Kiviranta I, Jurvelin JS (2002) Novel mechano-acoustic technique and instrument for diagnosis of cartilage degeneration. Physiol Meas 23: 491-503.

Laasanen MS, Saarakkala S, Töyräs J, Hirvonen J, Rieppo J, Korhonen RK, Jurvelin JS (2003a) Ultrasound indentation of bovine knee articular cartilage in situ. J Biomech 36: 1259-1267.

Laasanen MS, Töyräs J, Vasara AI, Hyttinen MM, Saarakkala S, Hirvonen J, Jurvelin JS, Kiviranta I (2003b) Mechano-acoustic diagnosis of cartilage degeneration and repair. J Bone Joint Surg Am 85-A Suppl 2: 78-84.

Laasanen MS, Saarakkala S, Töyräs J, Rieppo J, Jurvelin JS (2005) Site-specific ultrasound reflection properties and superficial collagen content of bovine knee articular cartilage. Phys Med Biol 50: 3221-3233.

Lammentausta E, Kiviranta P, Nissi MJ, Laasanen MS, Kiviranta I, Nieminen MT, Jurvelin JS (2006) T2 relaxation time and delayed gadolinium-enhanced MRI of cartilage (dGEMRIC) of human patellar cartilage at $1.5 \mathrm{~T}$ and 9.4 T: Relationships with tissue mechanical properties. J Orthop Res 24: 366-374.

Mankin MJ, Dorfman H, Lippiello L, Zarins A (1971) Biochemical and metabolic abnormalities in articular cartilage from osteo-arthritic human hips. II. Correlation of morphology with biochemical and metabolic data. J Bone Joint Surg Am 53: 523-537. 
Nieminen MT, Töyräs J, Rieppo J, Hakumäki JM, Silvennoinen J, Helminen HJ, Jurvelin JS (2000) Quantitative MR microscopy of enzymatically degraded articular cartilage. Magn Reson Med 43: 676-681.

Nieminen MT, Töyräs J, Rieppo J, Silvennoinen MJ, Hakumäki JM, Hyttinen MM, Helminen HJ, Jurvelin JS (2001) T2 relaxation reveals spatial collagen architecture in articular cartilage: a comparative quantitative MRI and polarized light microscopic study. Magn Reson Med 46: 487-493.

Nieminen MT, Rieppo J, Silvennoinen J, Töyräs J, JHakumaki JM, Hyttinen MM, Helminen MJ, Jurvelin JS (2002) Spatial assessment of articular cartilage proteoglycans with Gd-DTPA-enhanced T1 imaging. Magn Reson Med 48: 640-648.

Nieminen HJ, Saarakkala S, Laasanen MS, Hirvonen J, Jurvelin JS, Töyräs J (2004a) Ultrasound attenuation in normal and spontaneously degenerated articular cartilage. Ultrasound Med Biol 30: 493-500.

Nieminen MT, Töyräs J, Laasanen MS, Silvennoinen J, Helminen HJ, Jurvelin JS (2004b) Prediction of biomechanical properties of articular cartilage with quantitative magnetic resonance imaging. J Biomech 37: 321-328.

Nissi MJ, Töyräs J, Laasanen MS, Rieppo J, Saarakkala S, Lappalainen R, Jurvelin JS, Nieminen MT (2004) Proteoglycan and collagen sensitive MRI evaluation of normal and degenerated articular cartilage. J Orthop Res 22: $557-564$.

Palmoski MJ, Brandt KD (1981) Running inhibits the reversal of atrophic changes in canine knee cartilage after removal of a leg cast. Arthritis rheum 24: 1329-1337.

Pritzker KP, Gay S, Jimenez SA, Ostergaard K, Pelletier JP, Revell PA, Salter D, van den Berg WB (2006) Osteoarthritis cartilage histopathology: grading and staging. Osteoarthritis Cartilage 14: 13-29.

Radin EL (1976) Mechanical aspects of osteoarthrosis. Bull Rheum Dis 26: 862-865.

S Saarakkala, MS Laasanen, JS Jurvelin, K Torronen, MJ Lammi, R Lappalainen, J Töyräs (2003) Ultrasound indentation of normal and spontaneously degenerated bovine articular cartilage. Osteoarthritis Cartilage 11: 697 705 .

Saarakkala S, Töyräs J, Hirvonen J, Laasanen MS, Lappalainen R, Jurvelin JS (2004) Ultrasonic quantitation of superficial degradation of articular cartilage. Ultrasound Med Biol 30: 783-792.

Saarakkala S, Laasanen MS, Töyräs J, Jurvelin JS (2006) Quantitative ultrasound imaging detects articular cartilage and subchondral bone changes in spontaneous cartilage degeneration. Trans Orthop Res Soc 31: 227.

Shapiro EM, Borthakur A, Kaufman JH, Leigh JS, Reddy R (2001) Water distribution patterns inside bovine articular cartilage as visualized by $1 \mathrm{H}$ magnetic resonance imaging. Osteoarthritis Cartilage 9: 533-538.

Suh JK, Youn I, Fu FH (2001) An in situ calibration of an ultrasound transducer: a potential application for an ultrasonic indentation test of articular cartilage. J Biomech 34: 1347-1353.

Töyräs J, Laasanen MS, Saarakkala S, Lammi MJ, Rieppo J, Kurkijärvi J, Lappalainen R, Jurvelin JS (2003) Speed of sound in normal and degenerated bovine articular cartilage. Ultrasound Med Biol 29: 447-454.

Xia Y, Moody JB, Burton-Wurster N, Lust G (2001) Quantitative in situ correlation between microscopic MRI and polarized light microscopy studies of articular cartilage. Osteoarthritis Cartilage 9: 393-406.

Xia Y, Moody JB, Alhadlaq H (2002) Orientational dependence of $\mathrm{T} 2$ relaxation in articular cartilage: A microscopic MRI (microMRI) study. Magn Reson Med 48: $460-469$.

\section{Discussion with Reviewer}

M. Stoddart: When considering the clinical applicability of the arthroscopic methods described, do the authors believe that it is likely that any invasive technique, however minimal, will ever be adopted for the determination of early asymptomatic OA?

Authors: At present, each year hundreds of thousands of arthroscopies are performed to patients having problems with their knee. In addition to other pathologies such as meniscal tears, and ligament injuries, these patients may also have cartilage lesions, asymptomatic early OA or advanced OA. These patients seeking relief to their joint problems is the population that might benefit best of the information obtained with the described arthroscopic instrument. When the arthroscopy has been already planned to do, measurement with the instrument would not increase the operation risk more than the use of any other instrument during the operation. The obtained information could be used to study risk factors for OA development and, later, the results of such studies could help to predict OA risk as well as to apply appropriate procedures to prevent progression of OA changes. Also, direct mechanical measurements can help to judge objectively the results of cartilage repair techniques. Further, monitoring of tissue maturation after repair surgery will help the clinicians to determine optimal amount, pattern and time schedule of external stimulation, such as mechanical loading of the joint with repaired cartilage. For screening healthy, asymptomatic individuals, it is likely that low-cost non-invasive examinations, although not readily available, are needed. 